\title{
The patterns and risks for disease spreading of cattle movement in China
}

\author{
Xiangdong Sun ${ }^{1}$, Yongjun Liư ${ }^{1}$, Youming Wang ${ }^{1}$, Peng $\mathrm{Li}^{2}$, Aizhen $\mathrm{Guo}^{3}$, Zhining $\mathrm{Jia}^{1}$, \\ Xiaofeng Wang ${ }^{4}$, Hongjie Zhang ${ }^{5}$, Jian Zhang ${ }^{6}$, Yong Yu ${ }^{7}$, Yanwei Sun ${ }^{8}$, Baoxu Huang ${ }^{{ }^{*}}$ \\ ${ }^{1}$ China Animal Health and Epidemiology Center, Qingdao, China; ${ }^{*}$ Corresponding Author: huangbaoxu@hotmail.com \\ ${ }^{2}$ School of Public Health, Wuhan University, Wuhan, China \\ ${ }^{3}$ School of Animal Science and Technology, Huazhong Agricultural University, Wuhan, China \\ ${ }^{4}$ Jilin Province Center for Animal Disease Control and Prevention, Changchun, China \\ ${ }^{5}$ Shandong Province Center for Animal Disease Control and Prevention, Jinan, China \\ ${ }^{6}$ Henan Province Center for Animal Disease Control and Prevention, Zhengzhou, China \\ ${ }^{7}$ Sichuan Province Center for Animal Disease Control and Prevention, Chengdu, China \\ ${ }^{8}$ Guangdong Province Center for Animal Disease Control and Prevention, Guangzhou, China
}

Received 9 September 2013; revised 3 November 2013; accepted 16 November 2013

Copyright (C) 2013 Xiangdong Sun et al. This is an open access article distributed under the Creative Commons Attribution License, which permits unrestricted use, distribution, and reproduction in any medium, provided the original work is properly cited.

\begin{abstract}
In order to reduce the risks for the spread of disease in cattle movements, we investigated China's cattle breeding and movement pattern, analyzed risk factors of disease infection caused by long-distance movement, explored the relevant risk management measures and conjectured the direction of cattle movement based on the regional distribution of cattle inventory and beef price. We also constructed a market value chain in live cattle movements and qualitatively analyzed the risks for unapparent infection in the process of movement. Meanwhile, we put forward a long-term policy of reducing the risks for unapparent infection and animal health supervision measures.
\end{abstract}

Keywords: Cattle Movement; Disease Spread; Market Value Chain; Risk Analysis

\section{INTRODUCTION}

In recent years, with the development of animal husbandry and the improvement of people's living standards, as well as the implementation of various standardized cattle breeding programs in the national west development, animal movements become more frequent and the risks for the spread of animal disease get higher and higher. Canada, Britain, Denmark, Sweden and some other countries' research results showed that livestock and poultry movement plays an important role in the spread of animal disease. The researchers also suggested that in the management of animal movements we should pay attention to the following points: 1) Mixed degree, which means the mixed level of directly infected animals and indirectly infected animals; 2) Dispersion degree, that is, one buys infected animals from another seller, then the infected animals involved in trading become scattered in the whole group of animals; 3) Complicated sources degree, namely, the buyer will buy animals from a number of sources [1-8]. However, the relevant research in China is scarce [9].

Due to the lack of professional knowledge and effective means to assess disease risks in the process of cattle movements, most farmers can't catch the key points in disease prevention, and constantly introduce new disease or exogenous disease, which makes a serious threat to livestock health and huge loss to production $[1,7,9,10$ 12]. In order to reduce the risks for spread of disease in cattle movements, through describing the cattle markets and analyzing the risks in the process of cattle movements, this article wanted to provide the advice about the prevention of animal disease to farmers, practitioners and other related staffs in cattle movements and the reference to establish the disease spread mathematical model and dynamic model [3].

\section{MATERIALS AND METHODS}

\subsection{Central Location Survey}

The data of cattle price, including the price of cattle and buffalo specialized breeding or replaced for meat, was collected from the 336 national animal disease sentinel surveillance stations and border monitoring stations in China (the left of Figure 1). 

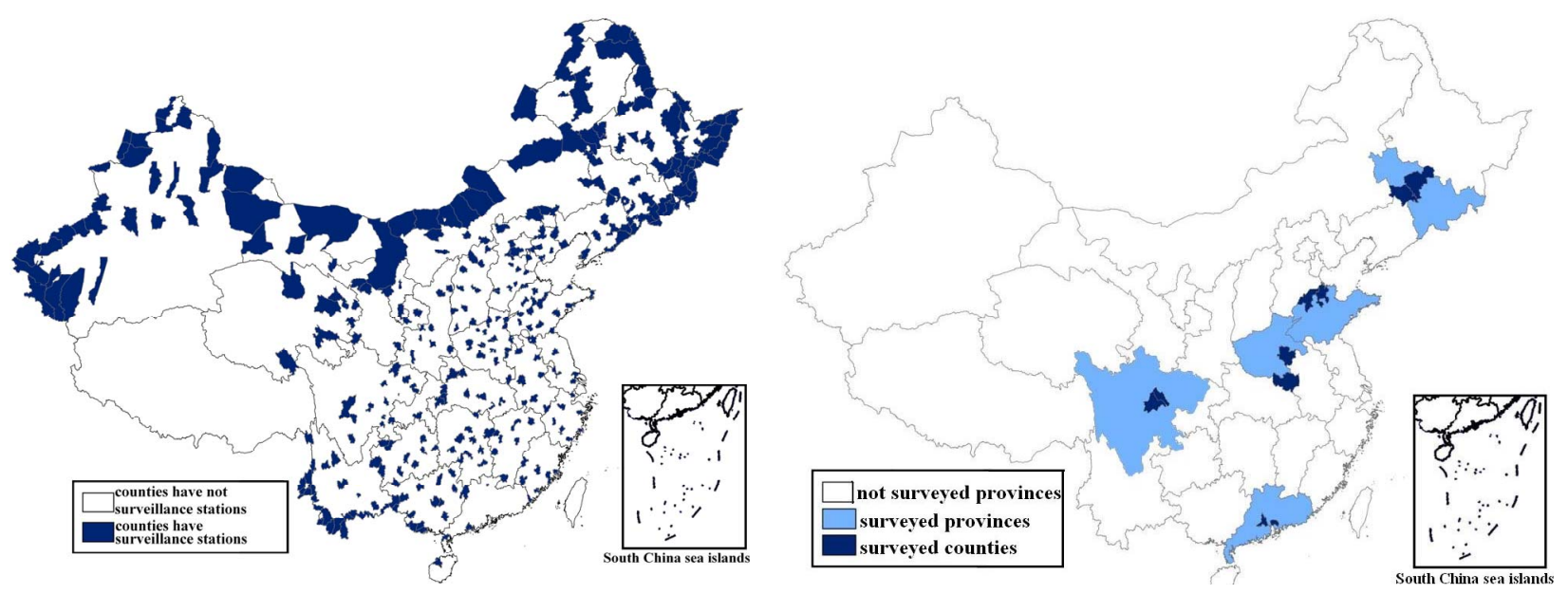

Figure 1. The regional distribution of samples (Left is the distribution of sentinel surveillance stations and border monitoring stations and right is the distribution of surveyed counties).

\subsection{Field Survey}

The cattle movement data was collected through field survey. We randomly drew 10 counties and chose 68 farms, 18 trading markets and 11 abattoirs in Jilin, Shandong, Henan, Sichuan and Guangdong provinces to describe the cattle movement pattern. The distribution of surveyed counties is shown as the right of Figure 1.

\subsection{Laboratory Test}

We chose cattle by stratified sampling method in 5 surveyed provinces and detected the cattle mycoplasma pneumonia by RT-PCR.

\section{RESULT AND DISCUSSION}

\subsection{Description of Cattle Movements}

\subsubsection{Regional Distribution of Cattle}

Most of cattle breeding in China were distributed in Northeast, North, Northwest and Southwest China, while the two highest breeding density regions were Northeast and North China, as shown in the Figure 2.

\subsubsection{Variance of Cattle Price}

The difference of cattle breeding stock's regional distribution leads to the difference of cattle's price to some extent. According to our survey, the price of killing cattle in Fujian provinces was highest, with an average of 27.6 $\mathrm{RMB} / \mathrm{kg}$, while the price in Hainan provinces was lowest, with an average of $11.7 \mathrm{RMB} / \mathrm{kg}$, and the former was 2.4 times higher than the latter. Figure 3 shows the regional distribution of killing cattle's price. By comparing Figure 3 with Figure 2, we got a tentative conclusion that the higher the density of cattle breeding, the lower the price of cattle in the same region. Correlation analysis showed that there was significant association between the local price of cattle and density of cattle breeding, and the Pearson product- moment correlation coefficient was -0.336 , which means a negative correlation exists between them.

\subsubsection{Directions of Cattle Movements}

According to the survey, the number of cattle transported across the provinces accounted for about $20 \%$ of the total trading volume. By comparing Figure 4 with Figure 3, we got the following conclusions. 1) The cattle's price contributed to their flow directions; 2) Most of the fattening cattle came from Northeast and North China and were transported to the other parts of the country; 3) Most of the adult cattle came from Northeast, Northwest and North China and were transported to Southeast China; 4) The principal cattle repository centers were distributed in Jilin, Liaoning, Shandong, Hebei and Henan provinces; 5) Jiangsu, Anhui, Zhejiang, Fujian, Guangdong provinces and other south-eastern provinces got adult cattle from the provinces mentioned in item 4 to slaughter and sell out.

\subsection{Analysis of Cattle Markets}

\subsubsection{Cattle Breeding and Sales}

The basic characteristics of cattle breeding in China are dispersed breeding, centralized fattening and the vast majority of calf being bred in backyard. According to statistics, the number of cattle stocks in backyard (less than 10 cattle) accounted for about two-thirds of the total cattle stocks, while small-scale farms (from 10 to 50 cattle) accounted for $1 / 6$ and commercial farms (more than 50 cattle) accounted for the rest, as shown in the Figure 5.

\subsubsection{Market Value Chain of Live Cattle Movement}

Our study showed that the market value chains of live 

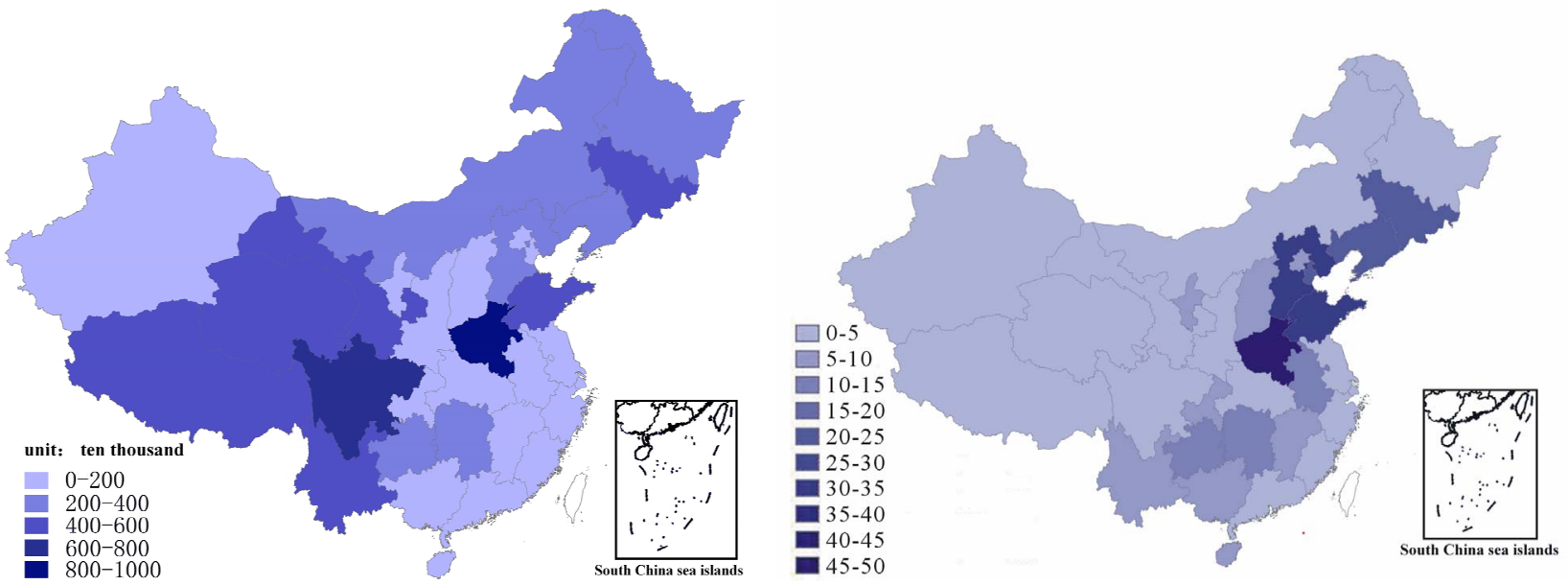

Figure 2. The distribution of cattle breeding stock and breeding density (left is the distribution of breeding stock and right is the distribution of breeding density at the end of 2007).

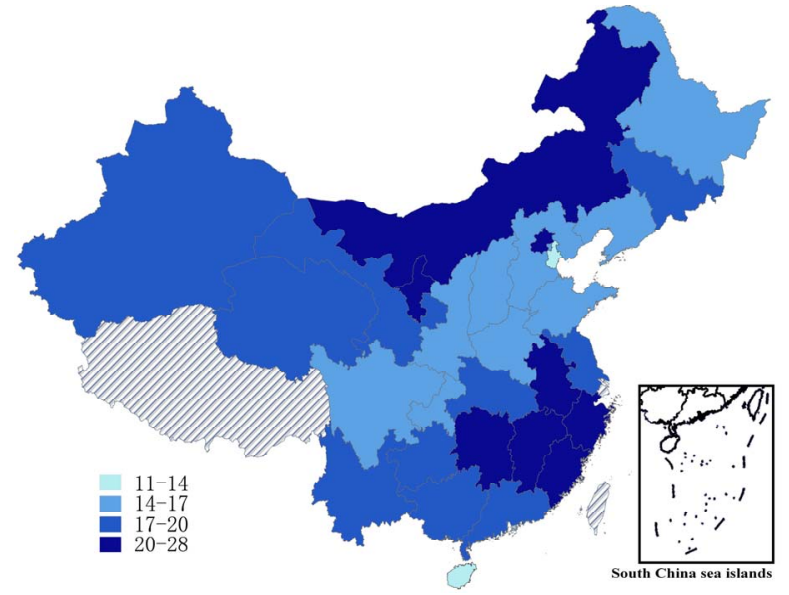

Figure 3. The distribution of killing cattle's price (do not include the data of Tibet Autonomous Region).
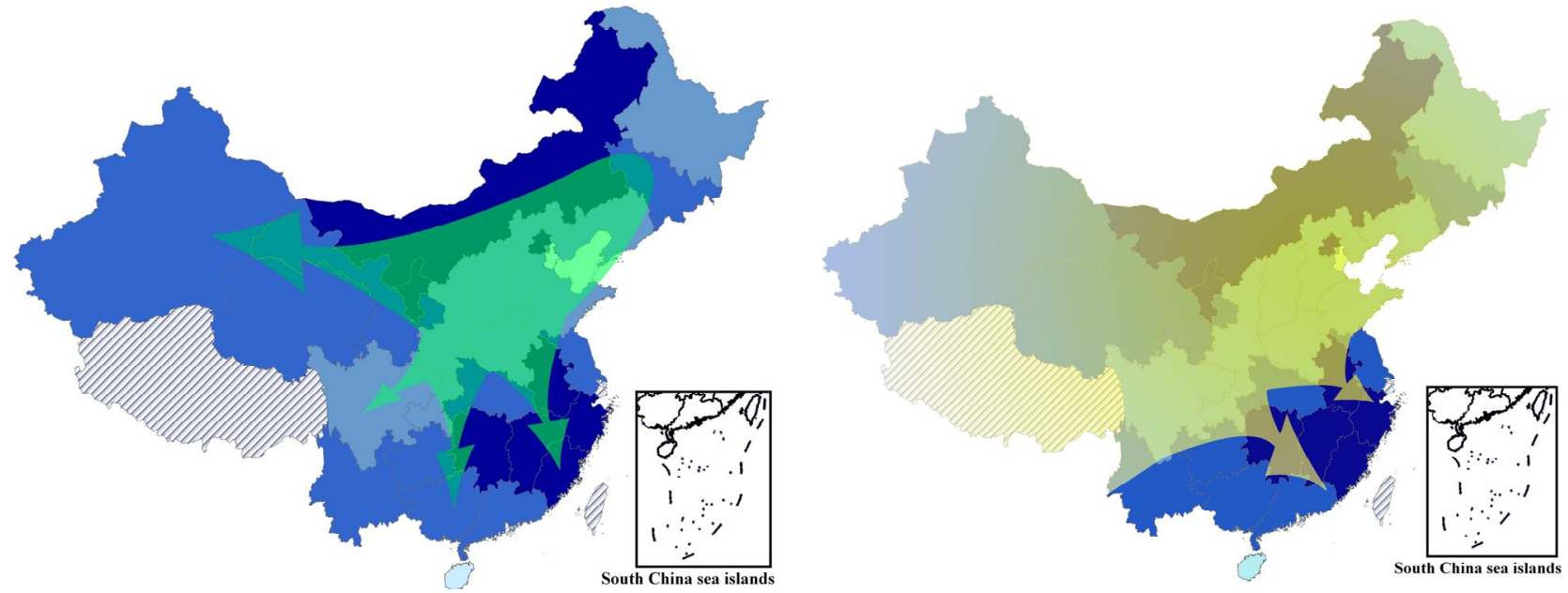

Figure 4. The directions of live cattle movements (left shows the directions of fattening cattle movements and right shows the directions of adult cattle movements, they do not include the data of Tibet Autonomous Region). 


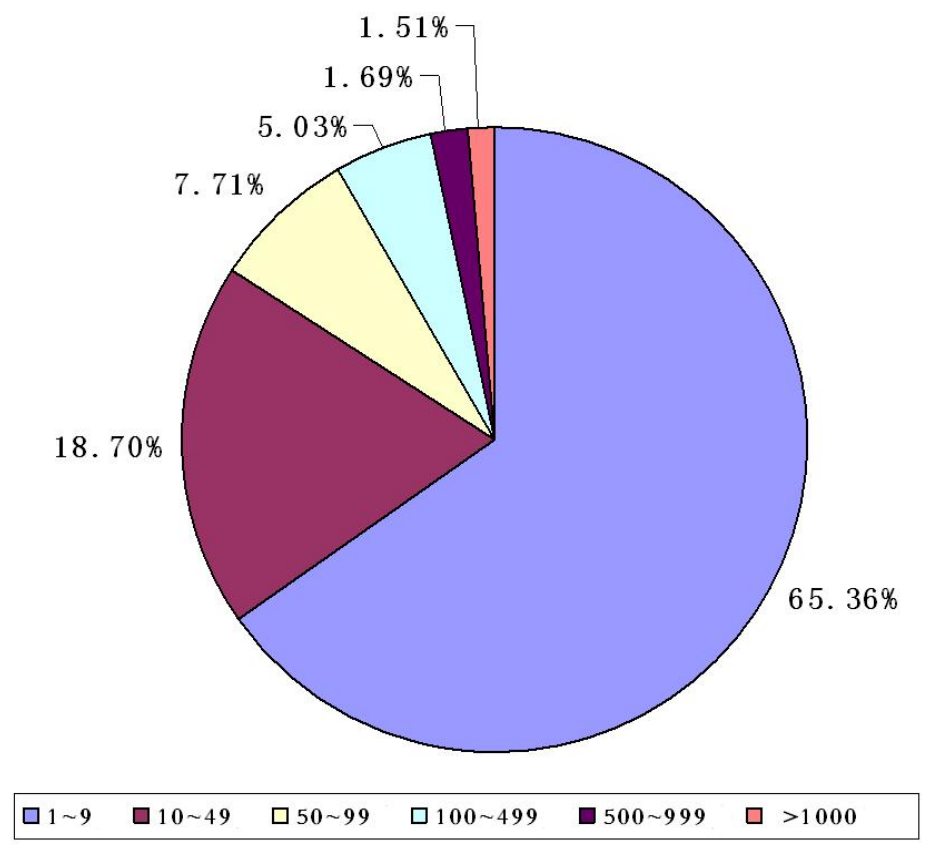

Figure 5. The proportions of different cattle stocks in farms.

cattle movement were complicated but regular, and the chains Mainly included two types: one was backyardmarket - fattening farm - abattoir, another is backyardmarket - backyard - market - abattoir. About $20 \%$ of the calves bred by in backyard were sold directly, $50 \%$ were sold when they reached the weight range from $150 \mathrm{~kg}$ to $350 \mathrm{~kg}$, and the rest sold when they were grown up. The fattening farms purchased calf or fattening cattle from markets, and then they sold out to abattoirs directly after fattening. The whole process of live cattle movement was showed in the Figures 6 and 7.

\subsubsection{Transaction in Live Cattle Markets}

The main characteristics of cattle transaction in China are sellers trading in live cattle markets and brokers participating in the process of buying and selling. According to our survey, about $20 \%$ of cattle sold once in their lifetime, $60 \%$ sold twice and the test sold more than 3 times. In other words, the total trading volume was 1.5 - 2 times larger than the actual number of slaughtered cattle. Large trading markets could influence the trading volume of several provinces, while farmers markets or small-scale trading markets only used to purchase and sell local cattle. In addition, the trading volume of the biggest market was more than 200,000 capita in the surveyed provinces. The cattle trading market and vehicle used to transport cattle were showed in Figure 8.

It was common that brokers participated in live cattle transactions and two main patterns included in this process. 1) They bought cattle from backyards and fattening farms, and then they sold out after short-term feeding; 2) They played a role of mediator in the process of buying and selling and charged brokerage fees when the deal succeeded. Besides, most of brokers had low educational level and had engaged in this work for a long period.

\subsection{Risk Analysis to Disease Infection in Cattle Movements}

There exist three risk factors in market value chain. 1) Initial risk, which contributes to infection in cattle movements; 2) Contact risk, mainly exists in the process of trading; 3) Mixed risk, which makes animal disease transmits more easily in the mixed flock.

\subsubsection{Initial Risk}

Most cattle are bred in backyard, where the disease prevention conditions are generally poor, biosafety measures are incomplete and recessive carriers abound. In addition, we did the serological test of cattle mycoplasma pneumonia for 19 cattle, and it showed that the individual positive rate of cattle mycoplasma pneumonia was $52 \%$, the group positive rate reached up to $89.5 \%$. In other words, there exists a high carrier risk in the process of backyard cattle trading.

\subsubsection{Contact Risk}

Contact risk mainly exists in three processes. 1) In the process of buying, shared vehicles or other transportations can carry pathogens; 2) In the process of short-term breeding, it makes direct transmission among cattle from different sources possible; 3 ) In the process of trading in the markets, these cattle from different sources can get cross-infection. In addition, the exposure risk exists in 


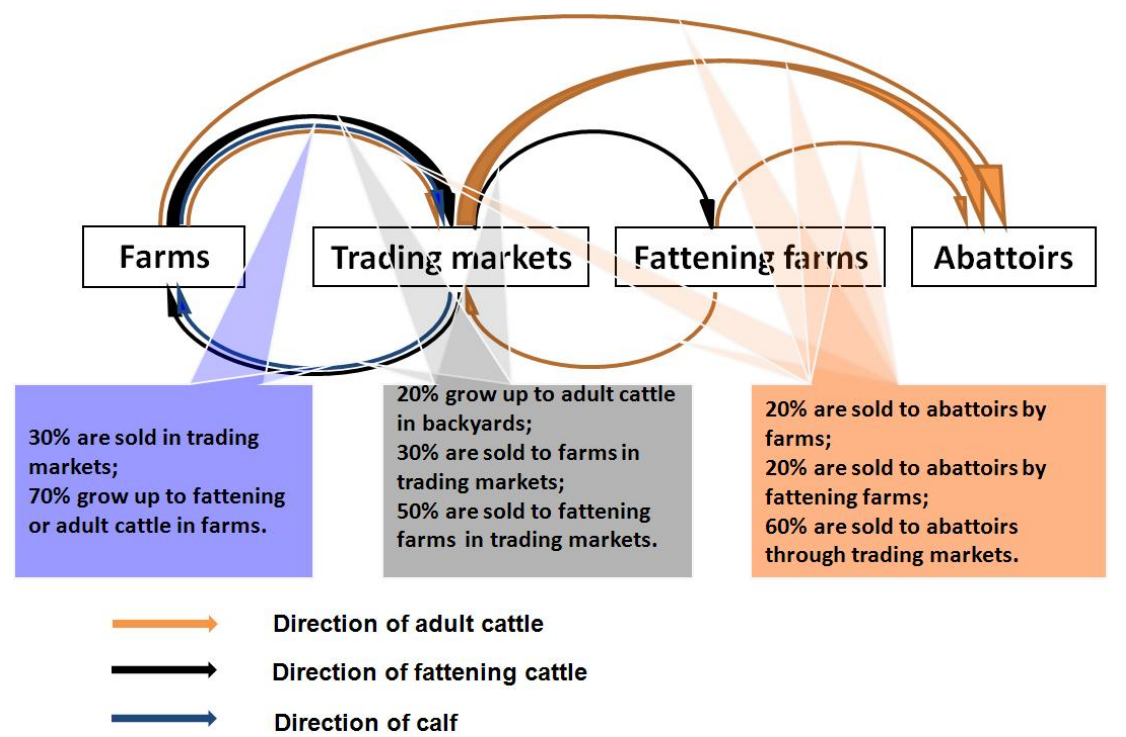

Figure 6. Market value chain of live cattle movement.

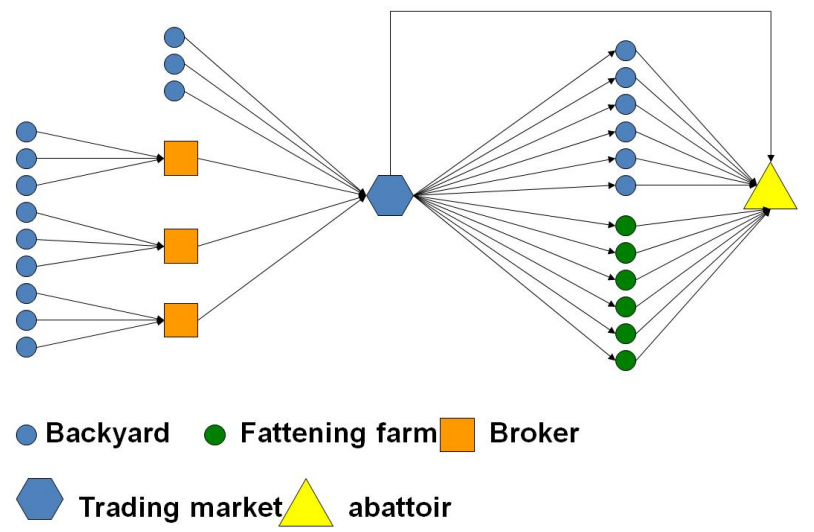

Figure 7. The process of flocks mixing in cattle movements.
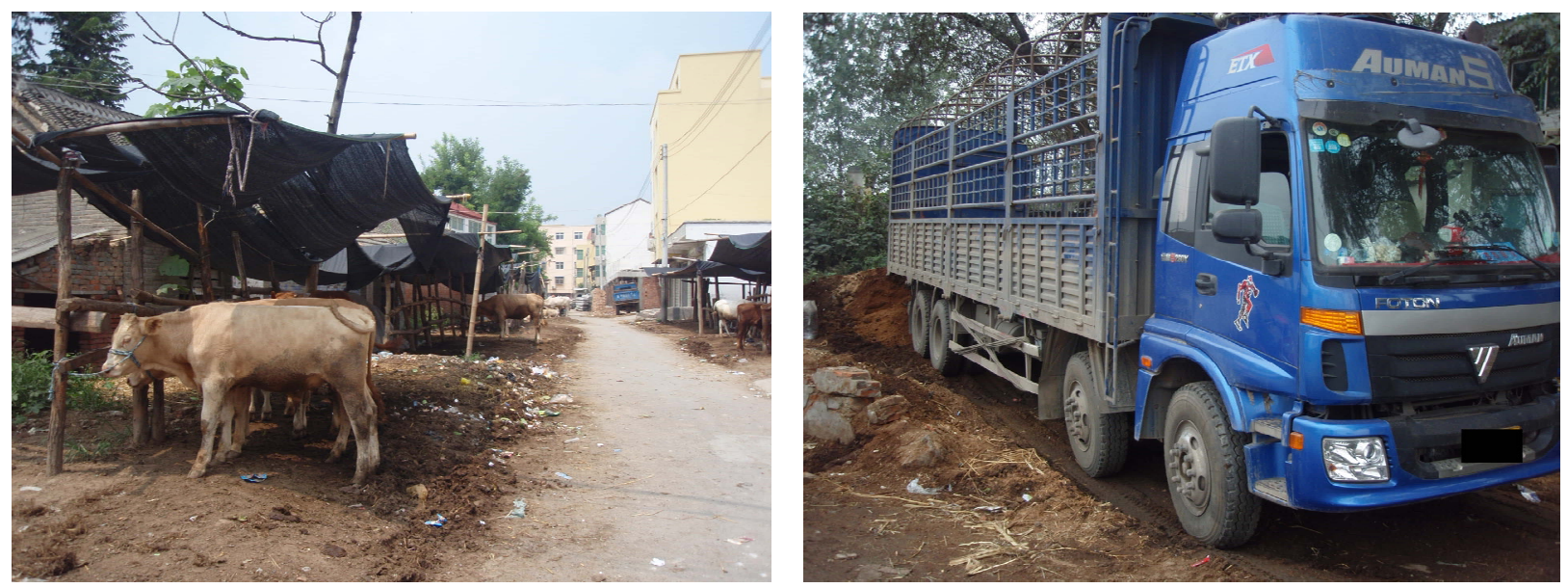

Figure 8. The trading market and transport (left is one of the live cattle trading markets we investigated and right is a common vehicle used to transport cattle).

the environment of all above processes. As we can see in Figure 9, under the condition of brokers' participation, it takes 15 days to accomplish a complete chain from cattle movement to mixed flock breeding, which is just as same 

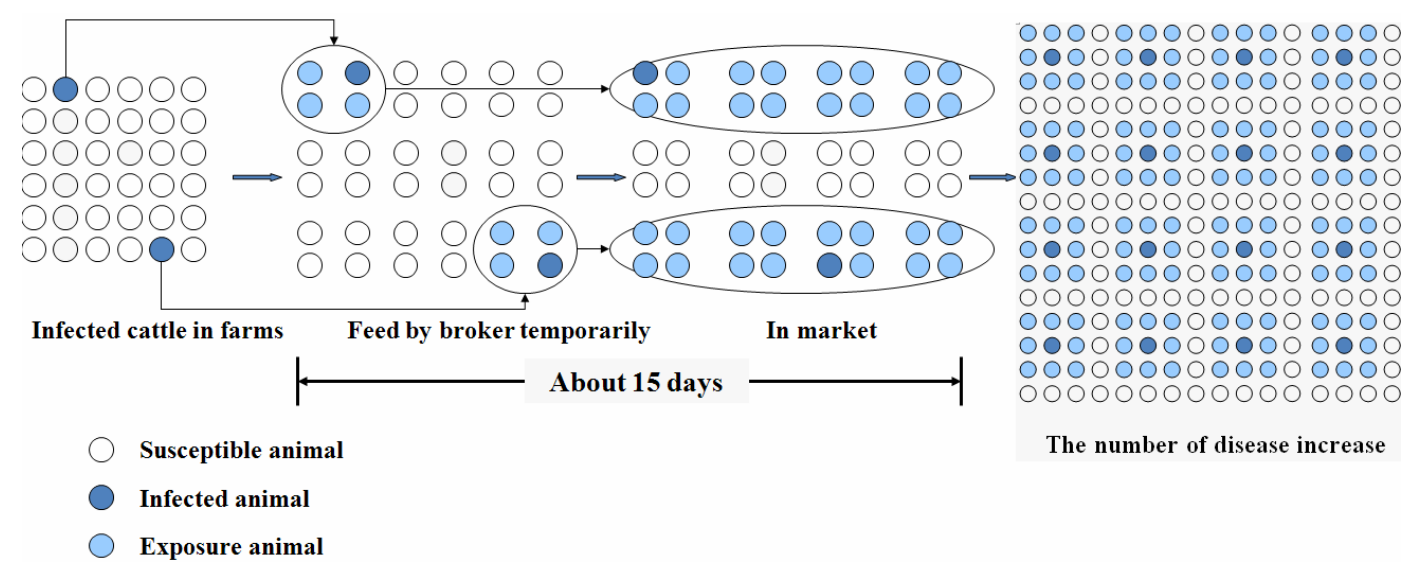

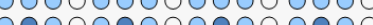
0 0 0 o o o o o o o

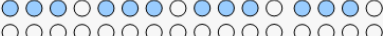
0000000000000000

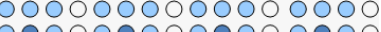
0000000000000000 0000000000000000 0000000000000000 0000000000000000 0000000000000000 0000000000000000

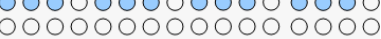

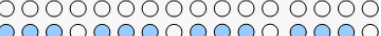

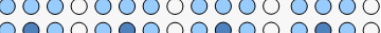

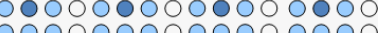
0000000000000000
0000000000000000

The number of disease increase

Figure 9. A model of risk of unapparent infection in live cattle market chain.

duration as the incubation period of the disease such as FMD. Some initial infected cattle will be attacked due to low immunity and a lot of cattle in the incubation period will carry the pathogens to make disease a long distance spread. The statistical results of our study showed that the onset of FMD was common in the process of animal movements, while the onset of cattle mycoplasma pneumonia was occasionally seen in the process of animal movements. Moreover, in the investigation period, we found cattle dead of disease in the markets.

\subsubsection{Mixed Risk}

In the market chain, all adult cattle are slaughtered and about $30 \%$ of calf and $80 \%$ of fattening cattle are resold for being bred again. The stress conditions, such as Long-distance transport, changes of environment and food in cattle movements, will increase the risk of infection. In addition, if farmers mix flocks directly without taking quarantine measures, the risk of the spread of disease will increase, too. Figure 9 visually shows the risk of unapparent infection in live cattle market chain.

\section{CONCLUSION}

By modeling the live cattle trading market value chain in China and analyzing the risk factors of animal disease spread in cattle movements, we draw the following conclusions. Backyards in high breeding density regions (such as Northeast and North China, etc.), all the brokers and large trading markets should be the key objects in the prevention and control of animal disease in cattle movements. 1) The vaccine immunization and disease surveillance of cattle in backyards should be strengthened, and backyards' breeding environment and the level of biological safety should be improved; 2) The general animal disease's prevention and control knowledge lecture should be held regularly among brokers to propagandize, educate and answer some common questions; 3) The behaviors of large trading markets should be regulated; 4)
No sharing vehicles and quarantine measures should be taken before flocks mixing.

\section{REFERENCES}

[1] Luo, C.B. and Luo, X.F. (2010) The construction of disease risk assessment in livestock movements. Guizhou Animal Husbandry and Veterinary Medicine, 34, 39-40.

[2] Dubé, C., Ribble, C. and Kelton, D. (2010) An analysis of the movement of dairy cattle through 2 large livestock markets in the province of Ontario, Canada. The Canadian Veterinary Journal, 51, 1254-1260.

[3] Dubé, C., Ribble, C., Kelton, D. and McNab, B. (2008) Comparing network analysis measures to determine potential epidemic size of highly contagious exotic diseases in fragmented monthly networks of dairy cattle movements in Ontario Canada. Transboundary and Emerging Diseases, 55, 382-392. http://dx.doi.org/10.1111/j.1865-1682.2008.01053.x

[4] Bigras-Poulin, M., Thompson, R.A., Chriel, M., Mortensen, S. and Greiner, M. (2006) Network analysis of Danish cattle industry trade patterns as an evaluation of risk potential for disease spread. Preventive Veterinary Medicine, 76, 11-39. http://dx.doi.org/10.1016/j.prevetmed.2006.04.004

[5] Bigras-Poulin, M., Barfod, K., Mortensen, S. and Greiner, M. (2007) Relationship of trade patterns of the Danish swine industry animal movements network to potential disease spread. Preventive Veterinary Medicine, 80, 143165. http://dx.doi.org/10.1016/j.prevetmed.2007.02.004

[6] Kao, R.R., Danon, L., Green, D.M. and Kiss, I.Z. (2006) Demographic structure and pathogen dynamics on the network of livestock movements in Great Britain. Proceedings of the Royal Society B: Biological Sciences, 273, 1999-2007. http://dx.doi.org/10.1098/rspb.2006.3505

[7] Robinson, S.E., Everett, M.G. and Christley, R.M. (2007) Recent network evolution increases the potential for large epidemics in the British cattle population. Journal of the Royal Society Interface, 4, 669-674. http://dx.doi.org/10.1098/rsif.2007.0214

[8] Christley, R.M., Robinson, S.E., Lysons, R. and French, 
N.P. (2005) Network analysis of cattle movements in Great Britain. In: Mellor, D.J., Russel, A.M. and Wood, J.L.N., Ed., Society for Veterinary Epidemiology and Preventive Medicine, Nairn, Inverness, Scotland, 30 March-1 April 2005, 234-244.

[9] Natale, F., Giovannini, A., Savini, L., Palma, D., Possenti, L., Fiore, G. and Calistri, P. (2009) Network analysis of Italian cattle trade patterns and evaluation of risks for potential disease spread. Preventive Veterinary Medicine, 92, 341-350. http://dx.doi.org/10.1016/j.prevetmed.2009.08.026

[10] Maria, N., Nina, H., Susanna, S.L., Ann, L. and Annie, J. (2011) Network analysis of cattle and pig movements in
Sweden: Measures relevant for disease control and risk based surveillance. Preventive Veterinary Medicine, 99, 78-90. http://dx.doi.org/10.1016/j.prevetmed.2010.12.009

[11] Christley, R.M., Pinchbeck, G.L., Bowers, R.G., Clancy, D., French, N.P., Bennett, R. and Turner, J. (2005) Infection in social networks: Using network analysis to identify high-risk individuals. American Journal of Epidemiology, 162, 1024-1031. http://dx.doi.org/10.1093/aje/kwi308

[12] Green, D.M., Kiss, I.Z. and Kao, R.R. (2006) Modeling the initial spread of foot-and-mouth disease through animal movements. Proceedings of the Royal Society B: Biological Sciences, 273, 2729-2735.

http://dx.doi.org/10.1098/rspb.2006.3648 